\section{Solar Sail Orbits at Artificial Sun-Earth Libration Points}

\author{
Hexi Baoyin* and Colin R. McInnes ${ }^{\dagger}$ \\ University of Strathclyde, \\ Glasgow, Scotland G1 IXJ, United Kingdom
}

\section{Introduction}

$\mathbf{S}$ NCE the pioneering works of Farquhar, ${ }^{1}$ Farquhar et al., ${ }^{2}$ Howell, ${ }^{3}$ Breakwell and Brown, ${ }^{4}$ and many others, libration point halo orbits in the restricted three-body problem have attracted a great deal of attention. Other researchers ${ }^{5,6}$ have investigated the dynamics associated with solar sails in the restricted three-body problem. In principle, solar sails provide new families of libration points (libration surfaces, in fact) everywhere inside regions connected to the classical libration points. These artificial equilibria have potential applications for future space physics and Earth observation missions.

In this Note a new family of solar sail orbits will be investigated in the sun-Earth circular restricted three-body problem. It will be shown that periodic orbits can be developed that are displaced above or below the plane of the restricted three-body system. Whereas traditional halo orbits are centered on the classical libration points, these new orbits are associated with artificial libration points. The orbits are retrograde, circular orbits with a period half that of the orbit period of the two primary masses of the problem. Numerical analysis of stability and controllability of the orbits shows that the orbits are unstable but completely controllable with both lightness number (sail areal density) and sail attitude.

\section{Displaced Orbits}

In this Note, the sun-Earth circular restricted three-body system is considered for a solar sail, with a dimensionless equation of motion adopted with its representation closely following the work of McInnes et al. ${ }^{6}$ Let the mass of the Earth be $\mu$ and the mass of the sun be $1-\mu$, where $\mu=3.036 \times 10^{-6}$ for the sun-Earth system. Then the equation of motion of the solar sail in a rotating frame of reference may be written as ${ }^{6}$

$$
\ddot{\boldsymbol{r}}+2 \boldsymbol{\omega} \times \dot{\boldsymbol{r}}+\nabla U=\boldsymbol{a}
$$

where $\boldsymbol{\omega}$ is the angular velocity vector of the rotating frame and $\boldsymbol{r}$ is the position vector of the solar sail relative to the center of mass of the two primaries. In the dimensionless representation, the angular velocity vector has unit magnitude and is directed along the $z$ axis, normal to the $x-y$ plane of the restricted three-body system. The effective potential function $U$ is defined as

$$
U=V+\Phi
$$

where $V$ is the gravitational potential function of the two primaries and $\Phi$ is the potential due to the rotation of the frame of reference, defined as

$$
\begin{gathered}
V=-\left[(1-\mu) / r_{1}+\mu / r_{2}\right] \\
\Phi=-\frac{1}{2}(\boldsymbol{\omega} \times \boldsymbol{r}) \cdot(\boldsymbol{\omega} \times \boldsymbol{r})
\end{gathered}
$$

The solar radiation pressure acceleration $\boldsymbol{a}$ is defined as

$$
\boldsymbol{a}=\beta\left[(1-\mu) / r_{1}^{2}\right]\left[\left(\boldsymbol{r}_{1} \cdot \boldsymbol{n}\right)^{2} / r_{1}^{2}\right] \boldsymbol{n}
$$

where $\beta$ is the lightness number of the solar sail and $\boldsymbol{n}$ is the sail unit normal vector. The sail lightness number is related to the sail areal density $\sigma$ by $\beta=1.53 / \sigma \mathrm{g} \cdot \mathrm{m}^{-2}$ (Ref. 6). Because the solar radiation pressure force can never be directed sunward, the sail attitude is constrained such that $\left(\boldsymbol{r}_{1} \cdot \boldsymbol{n}\right) \geq 0$. Therefore, we cannot obtain equilibrium solutions in the entire space and there are natural forbidden regions, detailed discussion of which can be found in Ref. 6 .

To obtain artificial equilibrium surfaces, previous studies have considered the case where $\ddot{\boldsymbol{r}}=\dot{\boldsymbol{r}}=0$ and so $\nabla U=\boldsymbol{a}$ (Ref. 6). However, because the sail attitude and lightness number can in principle be controlled in Eq. (1) (small variations in the sail area can trim $\beta$ ), it is possible that the sail attitude and lightness number are controlled so that

$$
\nabla U=\boldsymbol{a}
$$

everywhere on an orbit in the allowed region. Then the following simple equation of motion can be obtained from Eq. (1):

$$
\ddot{r}+2 \omega \times \dot{r}=0
$$

This represents an orbit that will always be moving on an equilibrium surface. Equation (7) is completely solvable, and its solutions can be written as

$$
\begin{gathered}
x(t)=[x(0)+\dot{y}(0) / 2]-[\dot{y}(0) / 2] \cos 2 t+[\dot{x}(0) / 2] \sin 2 t \\
y(t)=[y(0)-\dot{x}(0) / 2]+[\dot{x}(0) / 2] \cos 2 t+[\dot{y}(0) / 2] \sin 2 t \\
z(t)=\dot{z}(0) t+z(0)
\end{gathered}
$$

where (0) represents the initial conditions of the displacement and velocity of the solar sail in the rotating frame of reference.

In Eqs. (8), taking the appropriate initial values, some interesting orbits can be generated in the plane of the restricted three-body system, or parallel to the plane. Setting $z(0)=0$ and $\dot{z}(0)=0$, retrograde circular orbits in the system plane with a dimensionless period of $\pi$ (half-year) will be obtained. Similarly, when setting $\dot{z}(0)=0$ and $z(0) \neq 0$, one can obtain an orbit displaced in the $z$ direction. However, because of limitations on the sail lightness number and sail attitude control, orbits located near or on an equilibrium surface, or a place where the change in lightness number between neighboring equilibrium surfaces is small, are desirable. According to Ref. 6, 
Table 1 Orbit parameters

\begin{tabular}{lccc}
\hline \hline Orbit & $x_{0}$ & $r_{0}$ & $z_{0}$ \\
\hline 1 & 0.9856 & 0.001 & 0.01 \\
2 & 0.9866 & 0.001 & 0.01 \\
3 & 0.9876 & 0.001 & 0.01 \\
\hline \hline
\end{tabular}

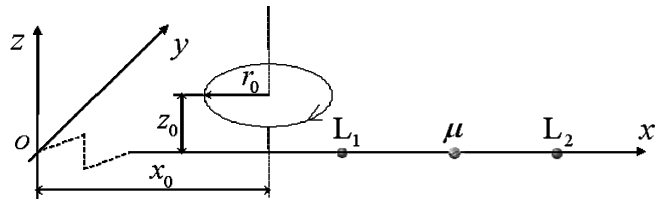

Fig. 1 Orbit illustration.

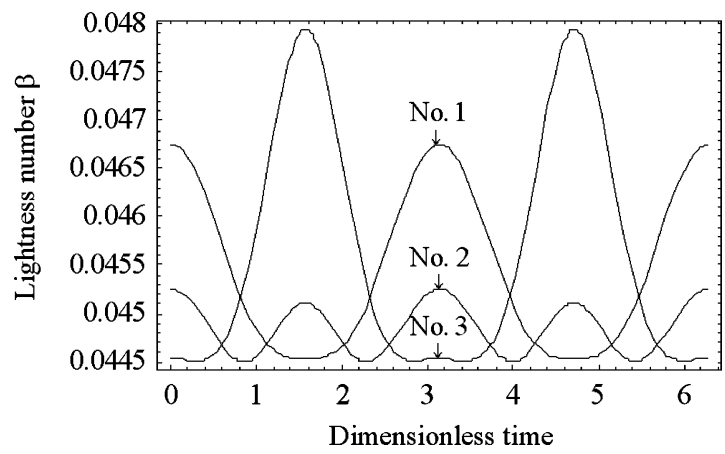

Fig. 2 Lightness number variations.

near the traditional libration points, the equilibrium surfaces are approximately elliptical and the change in lightness number between neighboring equilibrium surfaces is small. Therefore, these are suitable locations for achieving this family of periodic orbits, ensuring that the required variations in lightness number and sail attitude are small. Because the equilibrium surfaces are symmetric with respect to the $x-z$ plane, the center of this family of orbits should be located in this plane $\left(y_{0}=0\right)$.

The general process of designing this family of orbits first involves generation of the equilibrium surfaces in the vicinity of the required orbit using the method of Ref. 6 . Then, according to these equilibrium surfaces, the approximate orbit center is determined, which is usually taken near the center of an elliptical equilibrium surface. Following this process we designed several example orbits in the vicinity of $L_{1}$ (Fig. 1) with parameters (Table 1) where the position of $\mathrm{L}_{1}$ is $(0.989990864,0,0)$ calculated according to Ref. 7.

For the in-plane case, $z(0)=\dot{z}(0)=0$. If the other initial conditions in Eq. (8) are selected as $\dot{x}(0)=0$ and $y(0)=0$, circular orbits in the system plane with radius $r_{0}=|\dot{y}(0) / 2|$ and center $\left(x_{0}, y_{0}, z_{0}\right)=[x(0)+\dot{y}(0) / 2,0,0]$ will be obtained so that the orbit equations are

$$
x(t)=x_{0}-r_{0} \cos 2 t, \quad y(t)=r_{0} \sin 2 t
$$

It can be seen that the dimensionless orbit period is $\pi$ and that the orbit is retrograde relative to the rotating frame of reference. In the displaced case, $z(0)=z_{0} \neq 0$ and $\dot{z}(0)=0$. Similarly, we can obtain an orbit with center $\left(x_{0}, y_{0}, z_{0}\right)=[x(0)+\dot{y}(0) / 2,0, z(0)]$ and the same orbit equations.

In Figs. 2 and 3, the lightness number and sail attitude changes required for the orbits defined in Table 1 are given over an interval of two dimensionless orbit periods ( 1 year). These orbits are displaced approximately $1.5 \times 10^{6} \mathrm{~km}$ over $\mathrm{L}_{1}$ with radii of $150 \times 10^{3} \mathrm{~km}$. Orbit 2 is the most interesting, because the change in its lightness number is less than $2 \%$, and the change in sail pitch angle (angle of the sail normal relative to the sun line) is less than $8 \mathrm{deg}$. This means that the change in sail lightness number can be easily achieved in practice, perhaps using small articulated vanes to trim the effective sail area. This family of orbits can also be generated in the vicinity of the $\mathrm{L}_{2}$ point.

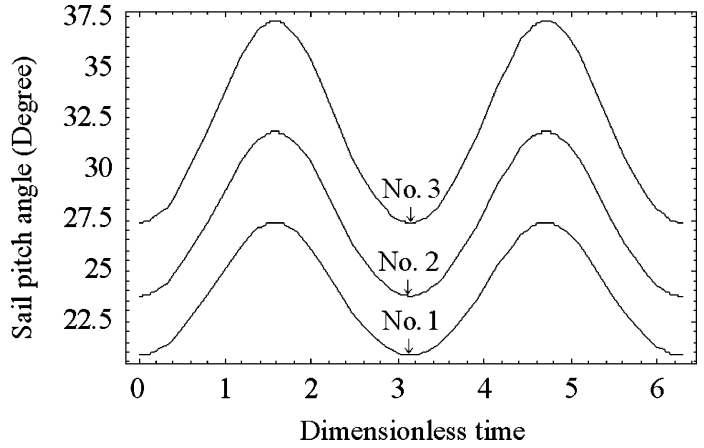

Fig. 3 Sail pitch angle variations.

\section{Orbit Stability and Control}

To determine the orbit stability in the linear sense, we apply an infinitesimal perturbation $\delta$ to the periodic orbit, and then the variation of Eq. (1) can be obtained:

$$
\ddot{\boldsymbol{\delta}}+2 \boldsymbol{\omega} \times \dot{\boldsymbol{\delta}}+\left(\frac{\partial(\nabla U)}{\partial \boldsymbol{r}}-\frac{\partial \boldsymbol{a}}{\partial \boldsymbol{r}}\right) \delta=0
$$

where the periodic orbit solution is substituted into the matrix

$$
K=\left[\frac{\partial(\nabla U)}{\partial \boldsymbol{r}}-\frac{\partial \boldsymbol{a}}{\partial \boldsymbol{r}}\right]
$$

and where

$$
\frac{\partial \boldsymbol{a}}{\partial \boldsymbol{r}}=\frac{2 \nabla U \nabla U^{T}}{\left(\boldsymbol{r}_{1} \cdot \nabla U\right)}-\frac{4 \nabla U \boldsymbol{r}_{1}^{T}}{\left(\boldsymbol{r}_{1} \cdot \boldsymbol{r}_{1}\right)}
$$

Because the matrix $K$ is time-dependent, the orbit stability in the linear sense becomes the stability of a linear system with timedependent coefficients. Equation (10) can be represented in the form of first-order differential equations as

$$
\dot{\varsigma}=A \varsigma
$$

where

$$
\varsigma=[\delta, \dot{\delta}]^{T}, \quad A=\left[\begin{array}{cc}
0 & I \\
-K & -S
\end{array}\right]
$$

$I$ is a unit matrix, and

$$
S=\left[\begin{array}{ccc}
0 & -2 & 0 \\
2 & 0 & 0 \\
0 & 0 & 0
\end{array}\right]
$$

Because the system has time-dependent coefficients, its stability cannot be directly determined by the eigenvalues of matrix $A$. Determining the stability of this system is much more difficult. However, there is a sufficient condition for asymptotic stability of time-dependent linear systems. ${ }^{8}$ If the eigenvalues of the matrix $H=\left(A+A^{T}\right) / 2$ are all negative, then the system defined by Eq. (12) is asymptotically stable. In this case,

$H=\frac{1}{2}\left\{\left[\begin{array}{cc}0 & I \\ -K & -S\end{array}\right]+\left[\begin{array}{cc}0 & I \\ -K & -S\end{array}\right]^{T}\right\}=\frac{1}{2}\left[\begin{array}{cc}0 & I-K^{T} \\ I-K & 0\end{array}\right]$

This is a real symmetric matrix; therefore, its eigenvalues all are real. On the other hand, from the special structure of the matrix $H$, it is not difficult to show that the eigenvalues of the matrix $\frac{1}{4}(I-K)^{T}(I-K)$ are the square of the eigenvalues of matrix $H$. Furthermore, the matrix $\frac{1}{4}(I-K)^{T}(I-K)$ also is a real symmetric matrix with real eigenvalues, and so half the eigenvalues of matrix $H$ must be positive. Therefore, the system defined by Eq. (12) cannot satisfy this asymptotically stable condition. For a periodic orbit, 
Table 2 Poles and control gains for orbit 2

\begin{tabular}{|c|c|c|c|c|c|c|}
\hline \multirow{2}{*}{$\frac{\text { Variable }}{\text { Desired poles }}$} & \multicolumn{6}{|c|}{ Values } \\
\hline & $-0.5+i$ & $-0.5-i$ & $-1+i$ & $-1-i$ & $-0.5+1.6 i$ & $-0.5-1.6 i$ \\
\hline Sail attitude control & {$\left[\begin{array}{c}47.2195 \\
-4.5786 \\
-82.516\end{array}\right.$} & $\begin{array}{l}-0.9030 \\
59.7843 \\
2.33290\end{array}$ & $\begin{array}{c}-9.6883 \\
1.14250 \\
40.6673\end{array}$ & $\begin{array}{l}9.74850 \\
-57.702 \\
-12.918\end{array}$ & $\begin{array}{l}19.2408 \\
27.8202 \\
-20.391\end{array}$ & $\left.\begin{array}{l}-1.0943 \\
0.80390 \\
57.6088\end{array}\right]$ \\
\hline Lightness number control & $G=[37.2837$ & -6.9285 & -0.8041 & 9.0263 & 11.9220 & $-6.0160]$ \\
\hline
\end{tabular}

however, because $A(t)=A(t+T)$, where $T$ is the period of the orbit, according to Floquet theory, the stability of a periodic coefficient linear system can be numerically determined. ${ }^{1}$ The basic idea is to first define a matrix $X(t)$ by

$$
\dot{X}(t)=A(t) X(t)
$$

where $X(0)=I$ is an appropriate dimensional unit matrix. Then Eq. (14) is numerically integrated to obtain $X(T)$. If the eigenvalues $\lambda_{i}$ of matrix $X(T)$ satisfy $\left|\lambda_{i}\right| \leq 1$, then the system is stable; otherwise, it is unstable. We have implemented the numerical simulation of numerous orbits, including the orbits presented in Table 1, and found that these orbits are all unstable. Therefore, in practice, the orbits must be controlled.

For a solar sail, lightness number control and sail attitude control are usually considered. Writing Eq. (12) with a linear control term yields

$$
\dot{\varsigma}=A \varsigma+B \boldsymbol{u}
$$

where, in the case of lightness number control,

$$
B=\left[\begin{array}{cccccc}
0 & 0 & 0 & \frac{\partial a_{x}}{\partial \beta} & \frac{\partial a_{y}}{\partial \beta} & \frac{\partial a_{z}}{\partial \beta}
\end{array}\right]^{T}
$$

$u=\delta \beta$, and $\boldsymbol{a}=\left[a_{x}, a_{y}, a_{z}\right]^{T}$. In the case of sail attitude control,

$$
B=\left[\begin{array}{ll}
0 & \frac{\partial \boldsymbol{a}}{\partial \boldsymbol{n}}
\end{array}\right]_{3 \times 6}^{T}
$$

where

$$
\frac{\partial \boldsymbol{a}}{\partial \boldsymbol{n}}=|\nabla U| I+\frac{2|\nabla U|}{\left(\nabla U \cdot \boldsymbol{r}_{1}\right)} \nabla U \boldsymbol{r}_{1}^{T}
$$

and $\boldsymbol{u}=\delta \boldsymbol{n}$, where matrices $A$ and $B$ are calculated on the periodic orbit. Because both $A$ and $B$ are time-dependent matrices, controllability of the time-varying system cannot be determined by the controllability matrix $Q=\left[\begin{array}{llllll}B & A B & A^{2} B & A^{3} B & A^{4} B & A^{5} B\end{array}\right]$. However, numerical investigation of the controllability of a particular orbit is possible. One can verify the controllability by verifying the rank of the controllability matrix $P$ of the system

$$
P\left(t, t_{0}\right)=\int_{t_{0}}^{t} \Phi\left(t_{0}, \tau\right) B(\tau) B^{T}(\tau) \Phi^{T}\left(t_{0}, \tau\right) \mathrm{d} \tau
$$

where $\Phi$ is the system transition matrix. The numerical results show that these three orbits are all completely controllable with both lightness number control and sail attitude control.

Next we will provide a simple linear feedback control scheme for orbit 2 , designed by a pole assignment technique. In this case, however, although matrix $A$ is time varying, from the special structure of this matrix and orbit parameters $\left(x_{0} \gg r_{0}\right)$ it is not difficult to show that the time-varying part of the matrix is relatively small. Representing matrices $A$ and $B$ as

$$
\begin{aligned}
& A(t)=A_{0}+\Delta_{A}(t) \\
& B(t)=B_{0}+\Delta_{B}(t)
\end{aligned}
$$

satisfies $\left\|A_{0}\right\| \gg\left\|\Delta_{A}(t)\right\|$ and $\left\|B_{0}\right\| \gg\left\|\Delta_{B}(t)\right\|$, where $A_{0}$ and $B_{0}$ are constant matrices. Taking advantage of these properties, a simple feedback control law can be designed. First we design the feedback gains for the system

$$
\dot{\boldsymbol{\varsigma}}=A_{0} \varsigma+B_{0} \boldsymbol{u}
$$

where full state feedback is assumed to be defined by

$$
\boldsymbol{u}=G \varsigma
$$

and where gain matrix $G$ will be obtained by pole assignment. The desired poles and designed gains are given in Table 2. Finally, the stability of the controlled system has to be numerically checked by using the aforementioned Floquet theory, because these gains are not exactly designed for the original system defined by Eq. (15), but for the approximate system defined by Eq. (19). The numerical results show that the controlled system is stabilized with these gains.

\section{Conclusions}

A family of displaced solar sail orbits near the sun-Earth libration points are presented in this Note. These orbits are either displaced over the $\mathrm{L}_{1}$ or $\mathrm{L}_{2}$ points and parallel to system plane or are in the system plane and near the libration points. Several particular orbits are designed and their stability and controllability are discussed by means of numerical methods. The results indicate that this family of periodic orbits is usually unstable but completely controllable by use of the sail attitude or lightness number control.

\section{References}

${ }^{1}$ Farquhar, R. W., "The Control and Use of Libration-Point Satellite," NASA TR R-346, Sept. 1970.

${ }^{2}$ Farquhar, R. W., Muhonen, D. P., Newman, C. R., and Heuberber, H. S., "Trajectories and Orbital Maneuvers for the First LibrationPoint Satellite," Journal of Guidance and Control, Vol. 3, No. 6, 1980, pp. 549-554.

${ }^{3}$ Howell, K. C., "Three-Dimensional, Periodic, 'Halo' Orbit," Celestial Mechanics, Vol. 32, April 1984, pp. 53-71.

${ }^{4}$ Breakwell, J. V., and Brown, J. V., "The 'Halo' Family of 3-Dimensional Periodic Orbits in the Earth-Moon Restricted 3-Body Problem," Celestial Mechanics, Vol. 20, Nov. 1979, pp. 389-404.

${ }^{5}$ McInnes, C. R., Solar Sailing: Technology, Dynamics and Mission Applications, Springer Praxis, London, 1999.

${ }^{6}$ McInnes, C. R., Mcdonald, A. J. C., Simmon, J. F. L., and MacDonald, E. W., "Solar Sail Parking in Restricted Three-Body System," Journal of Guidance, Control, and Dynamics, Vol. 17, No. 2, 1994, pp. 399-406.

${ }^{7}$ Szebehely, V., Theory of Orbits: The Restricted Problem of Three Bodies, Academic Press, New York, 1967.

${ }^{8}$ Afanasev, V. N., Kolmanovskii, V. B., and Nosov, V. R., Mathematical Theory of Control System Design, Kluwer Academic, London, 1996. 